\title{
Determination of Critical N-Factors for the CRM-NLF Wing
}

\author{
Normann Krimmelbein ${ }^{1(\bowtie)}$ and Andreas Krumbein ${ }^{2}$ \\ ${ }^{1}$ DLR, Institut für Aerodynamik und Strömungstechnik, Lilienthalplatz 7 , \\ 38108 Braunschweig, Germany \\ normann. krimmelbein@dlr.de \\ 2 DLR, Institut für Aerodynamik und Strömungstechnik, Bunsenstr. 10, \\ 37073 Göttingen, Germany
}

\begin{abstract}
Temperature sensitive paint images from a wind tunnel campaign for the CRM-NLF geometry are used to extract experimental transition lines to be used to determine critical $\mathrm{N}$-factors based on incompressible and compressible linear stability theory. Based on the $\mathrm{e}^{\mathrm{N}}$-method, transition locations are subsequently predicted for different flow conditions using RANS computations and the results are compared to the experiment.
\end{abstract}

Keywords: Critical N-factor · Transition prediction · Incompressible linear stability theory $\cdot$ Compressible linear stability theory

\section{Introduction}

To verify and validate a design method for airfoil shapes that lead to pressure distributions delaying transition on wings with large sweep angles and at high Reynolds number flows (CATNLF, Crossflow Attenuated Natural Laminar Flow [1]) a wind tunnel test campaign in the National Transonic Facility (NTF) at the NASA Langley Research Center has been conducted [2]. The experiments, also aiming at evaluating best practices for laminar flow testing and the general suitability of the National Transonic Facility for laminar flow investigations, where carried out for the flow around a semi-span model of the Common Research Model with Natural Laminar Flow (CRM-NLF). Experimental transition locations have been documented during the tests using temperature sensitive paint (TSP) images by applying different methods, namely a temperature step method and a heating layer approach [3]. The experimental results for the Mach number $\mathrm{M}=$ 0.856 and the Reynolds number $\operatorname{Re}_{\mathrm{MAC}}=15 \times 10^{6}$ based on the mean aerodynamic chord (MAC) at the angles of attack $\alpha=1.5^{\circ}, 2.0^{\circ}, 2.5^{\circ}$, and $3.0^{\circ}$ are used in the present work to extract transition lines for the different flow conditions and to determine critical $\mathrm{N}$-factors for the CRM-NLF wing. 


\section{Approach}

The purpose of this paper is to provide an answer to one of the many questions that arose in the discussions around the CATNLF approach, its suitability for an effective NLF design of a long-range transport aircraft wing with large sweep and the question if it is appropriate to execute the necessary LST analysis in a compressible manner as was done in the underlying approach the CRM-NLF wing design is based on. Compressible LST is favored by NASA [2] and Boeing [5], whereas incompressible LST is the trusted approach at DLR [4] and Airbus. One major point in the discussions which were held mainly on workshops and conferences in the US was the question if the execution of a compressible LST analysis (in contrast to an incompressible analysis) is needed so that an appropriate CATNLF design as achieved for the CRM-NLF wing can be found in the design process. A prerequisite for answering this question is the investigation of how the execution of compressible and incompressible LST analysis might affect the outcome of predicted transition lines for a given configuration given that the correspondingly calibrated critical $\mathrm{N}$-factors are available and consistently applied in the LST analyses. In this respect, the calibration of the critical N-factors is an aspect of major importance in this paper. In contrast to the data from $[2,5]$ in the present approach also critical Tollmien-Schlichting N-factors from incompressible LST are presented for the NTF, data that have not been published before. In addition, in the present paper the calibration process is described in detail.

To support and extend the existing evaluation of the critical Tollmien-Schlichting and cross flow N-factors for the CRM-NLF at the National Transonic Facility [2, 5], in the present work transition lines have been extracted from TSP images for different angles of attack and stability computations using incompressible and compressible linear stability theory (LST) have been carried out.

The temperature sensitive paint (TSP) images have been obtained during the test campaign in the National Transonic Facility at the NASA Langley Research Center using different transition detection approaches and image-enhancement methods [3]. For transition detection a heating layer approach has been used which has been supported by the results of a more traditional temperature step method. Due to a reduced contrast in the images, a Contrast Limited Adaptive Histogram Equalization technique has been applied to the images of the heating layer approach [3]. Transition lines have been determined by visual inspection of the experimental TSP images from both transition detection approaches. For this, the temperature step and heating layer images have been manually adjusted in terms of, for example, color levels and brightness to identify a clear as possible transition front. The parts of the transition front that have been determined with a certain amount of confidence have been connected by linear interpolation to give a continuous transition line. Interpolation was in particular needed for the unclear transition situation downstream of turbulent wedges or in regions where a lesser contrast of the TSP images prevented a clear identification of the transition front.

The stability computations are based on LST in form of the $\mathrm{e}^{\mathrm{N}-}$-method, utilizing the DLR TAU code, DLR's compressible unstructured RANS solver for external flows, and its transition prediction capabilities [4]. Transition prediction in the TAU code is realized through an automated process chain as outlined in detail in [4] consisting of the RANS solver, a procedure for providing the laminar boundary-layer data and a fully-automated 
LST code. The boundary-layer data are either provided by a laminar boundary-layer code or extracted directly from the RANS grid in cases where the boundary-layer code cannot provide data, for example, downstream of transonic shocks. The prediction of transition onset locations is realized using the $\mathrm{e}^{\mathrm{N}}$-method and a standard 2-N-factor approach applying critical N-factors for Tollmien-Schlichting (T-S) waves, $\mathrm{N}_{\mathrm{TS}}$, and crossflow (CF) vortices, $\mathrm{N}_{\mathrm{CF}}$. For the computation of incompressible and compressible amplification rates and $\mathrm{N}$-factors the prescribed-frequency/prescribed-propagation-direction $(\psi$ $=0^{\circ}$ ) integration strategy for the calculation of Tollmien-Schlichting amplifications and the prescribed-frequency $(\mathrm{F}=0 \mathrm{~Hz}) /$ prescribed-wavelength integration strategy for the calculation of cross-flow amplifications is used. That is, the standard $\mathrm{N}_{\mathrm{TS}}-\mathrm{N}_{\mathrm{CF}} 2-\mathrm{N}$ factor approach is applied assuming the highest T-S amplification occur for T-S waves propagating in the direction of the inviscid flow at the boundary-layer edge and stationary CF amplifications as the highest amplified cross-flow vortices. In a first step, critical $\mathrm{N}$-factors have been determined based on boundary-layer code computations along linein-flight cuts of the wing at different angles of attack. To verify the critical $\mathrm{N}$-factors, in a second step computations with automatic transition prediction have been performed, now with boundary layer profiles for the stability analysis directly extracted from the RANS solution.

A $30 \times 10^{6}$ nodes unstructured computational grid [7] has been used for all computations, with a resolution of the wing surface of approximately 450 grid points in span-wise direction and approximately 300 grid points in streamwise direction. The boundary layer above the wing surface is resolved with 90 structured layers with an initial spacing leading to $\mathrm{y}^{+}$values below 0.5 [7]. For the turbulent parts of the flow the negative Spalart-Allmaras (SA-neg) one-equation turbulence model is applied.

\section{Approach}

The extracted transition lines (solid yellow and orange lines in Figs. 3, 4, 5 and 6), which are based on the basic visual inspection of the TSP images, are extended by linear interpolations (dashed yellow and orange lines in Figs. 3, 4, 5 and 6) at span-wise locations where the actual natural transition front is concealed by turbulent wedges or the contrast in the TSP images complicate a clear identification of the transition front. The wing span of $\mathrm{s}=1.344 \mathrm{~m}$ extends from $\mathrm{y}=0.185 \mathrm{~m}$ to $\mathrm{y}=1.529 \mathrm{~m}$. As anticipated, both extracted transition lines, from temperature step method (yellow lines in Figs. 3, 4, 5 and 6) and from heating layer method (orange lines in Figs. 3, 4, 5 and 6), are very similar in location and also match those transition locations derived by other authors [2, 6] (magenta lines in Figs. 3, 4, 5 and 6).

Evaluation of the $\mathrm{N}$-factor for Tollmien-Schlichting instabilities at the extracted transition lines reveals an averaged critical Tollmien-Schlichting $\mathrm{N}$-factor of $\mathrm{N}_{\mathrm{TS}}=$ 11.7 for an incompressible stability analysis and a value of $\mathrm{N}_{\mathrm{TS}}=4.1$ for a compressible stability analysis (Fig. 1). In addition to the considerable scatter of the predicted $\mathrm{N}$-factors that occurs along the whole wing span and which is very typical for the calibration of critical $\mathrm{N}$-factors based on measurement data there are further uncertainties in the results in the span region with $\mathrm{y}>1 \mathrm{~m}$ (Fig. 1). Here, the evidently clearly identifiable transition fronts often correspond to the shock fronts on the wing, which slightly deviate 
from the experiment for the computations. This leads to the additional uncertainties in the predicted $\mathrm{N}$-factors (for $\mathrm{y}>1 \mathrm{~m}$ ) when either the shock location in the experiment is located further upstream compared to the CFD computation, resulting in lower values of the $\mathrm{N}$-factor, or the shock location in the experiment is located further downstream compared to the CFD computation, resulting in no values for the $\mathrm{N}$-factor because transition is not detected upstream of the shock. The shock fronts along the wing span can be identified by the grey-shade change from dark to light grey in the left and middle depictions of Figs. 3, 4, 5, and 6 starting at the wing root at about $\mathrm{x} / \mathrm{c}=0.75$. For the angles of attack $\alpha=1.5^{\circ}$ and $2.0^{\circ}$ in Fig. 3 and Fig. 4 a double-shock system exists with the second shock occurring on the outboard wing along the range from about $75 \%$ to about $90 \%$ of the wing span.

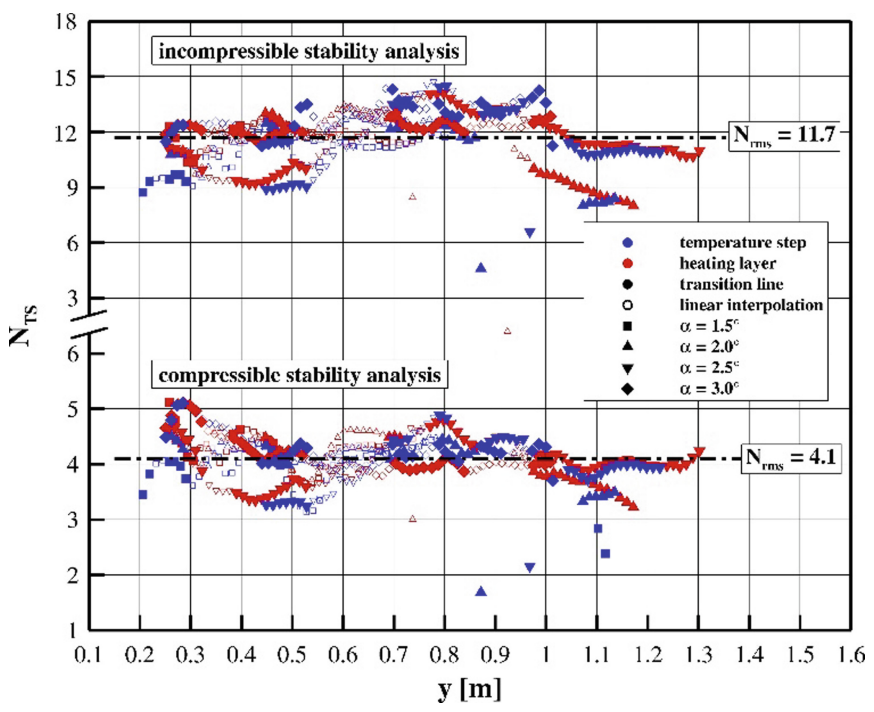

Fig. 1. CRM-NLF wing, Tollmien-Schlichting N-factors at transition along the wing span.

The present circumstance is caused by the application of the boundary-layer code for the computation of the boundary-layer profiles for the stability analysis, which does not provide a solution for separated flows (i.e. a short distance downstream of the strong pressure increase due to the shock). Comparing the compressible stability analysis results, the predicted critical Tollmien-Schlichting N-factors at transition are somewhat lower than used in other stability investigations [2] $\left(\mathrm{N}_{\mathrm{TS}}=4.1 \mathrm{vs} . \mathrm{N}_{\mathrm{TS}}=6.0\right)$ but are still just within the range of critical Tollmien-Schlichting $\mathrm{N}$-factors determined for the flow quality of the National Transonic Facility [5] $\left(\mathrm{N}_{\mathrm{TS}}=4.0-8.0\right)$.

Significant cross-flow amplifications that may lead to transition are only present for the lowest angle of attack of $\alpha=1.5^{\circ}$. Here, a value of approximately $\mathrm{N}_{\mathrm{CF}}=6.0$ is attained for incompressible as well as compressible stability analysis in the mid-span and outboard region of the wing (Fig. 2). However, whenever the cross-flow $\mathrm{N}$ factor of $\mathrm{N}_{\mathrm{CF}}$ $=6.0$ is reached also the critical Tollmien-Schlichting N-factor is reached (mid-span, Fig. 2) or would be reached a very short distance further downstream due to the existence 

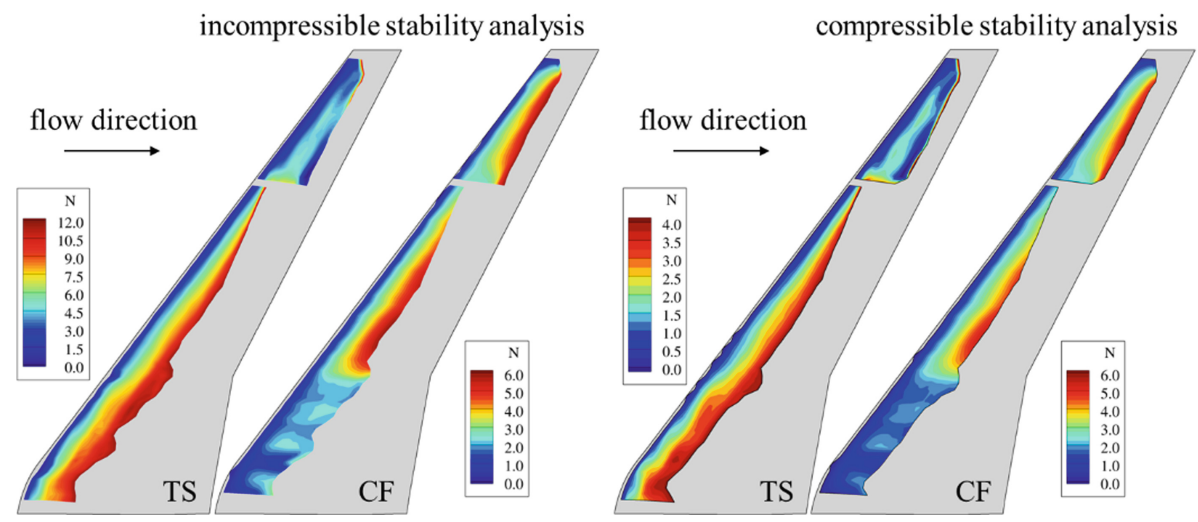

Fig. 2. CRM-NLF wing, incompressible (left) and compressible (right) Tollmien-Schlichting (TS) and cross-flow (CF) N-factors upstream of predicted transition locations, $\alpha=1.5^{\circ}$.

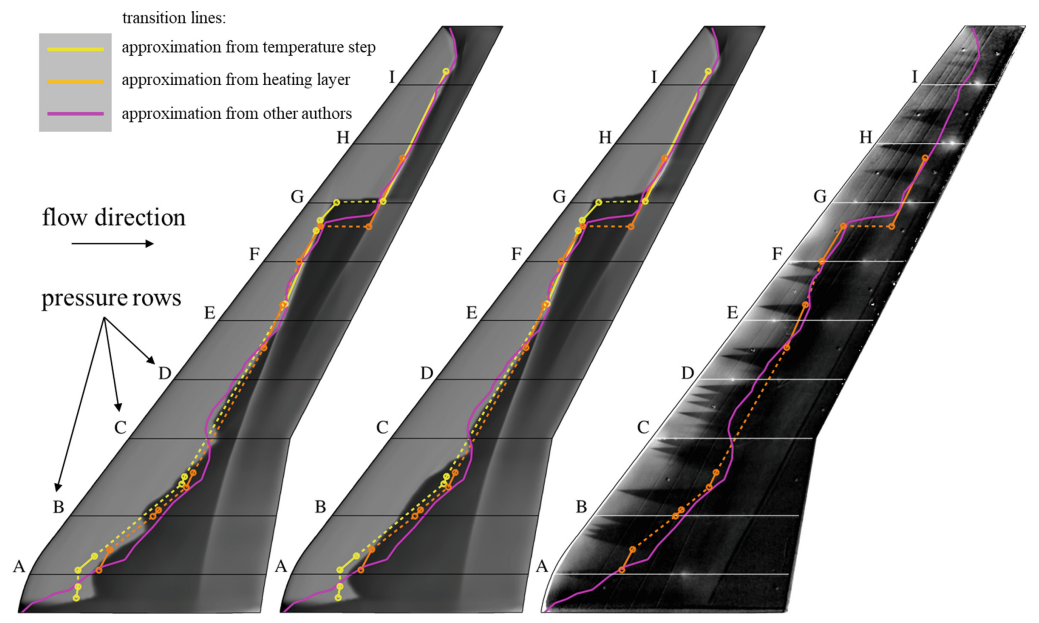

Fig. 3. CRM-NLF wing, comparison of predicted transition fronts, $\alpha=1.5^{\circ}$. Left: RANS surface $\mathrm{c}_{\mathrm{f}}$-distribution, incompressible LST. Middle: RANS surface $\mathrm{c}_{\mathrm{f}}$-distribution, compressible LST. Right: TSP image, heating layer method.

of a shock (mid-span and outboard, not shown here, visible for computations where cross-flow transition is not taken into account). As a result, computations with both, Tollmien-Schlichting and cross-flow amplifications considered or computations only taking into account stream-wise instabilities deliver nearly indistinguishable transition lines, leaving it open, if cross-flow instabilities play a role for $\alpha=1.5^{\circ}$. For all other angles of attack considered here the cross-flow $\mathrm{N}$-factor never reaches $\mathrm{N}_{\mathrm{CF}}=6.0$.

Looking at the predicted transition locations in Figs. 3, 4, 5, and 6 and comparing incompressible (left) with compressible (middle) stability results, it can be seen, that, despite the large difference in critical $\mathrm{N}$-factor, the numerical results are very similar for both methods. Laminar and turbulent regions of the CFD computations are visualized 
in these figures by the skin friction distribution from the RANS simulations, with light grey areas corresponding to low values of the skin friction coefficient, and hence to laminar flow, and dark grey areas corresponding to higher values of the skin friction coefficient, and hence to turbulent flow. Significant differences between the predicted transition fronts from incompressible and compressible stability analysis can be seen for $\alpha=2.0^{\circ}$ (Fig. 4) at the outboard section near the wing tip (around pressure row I) where with incompressible theory the extent of laminar flow is increased compared to compressible theory. Another difference can be seen for $\alpha=2.5^{\circ}$ (Fig. 5) at the midspan region between pressure rows $\mathrm{D}$ and $\mathrm{F}$, where a larger extent of laminar flow is achieved for the compressible approach. As already partly visible for $\alpha=2.5^{\circ}$ for $\alpha=$ $3.0^{\circ}$ (Fig. 6) with incompressible stability analysis transition is predicted upstream of the shock location in the outboard region of the wing (pressure rows F-I), whereas for compressible stability theory transition is predicted at the shock location.

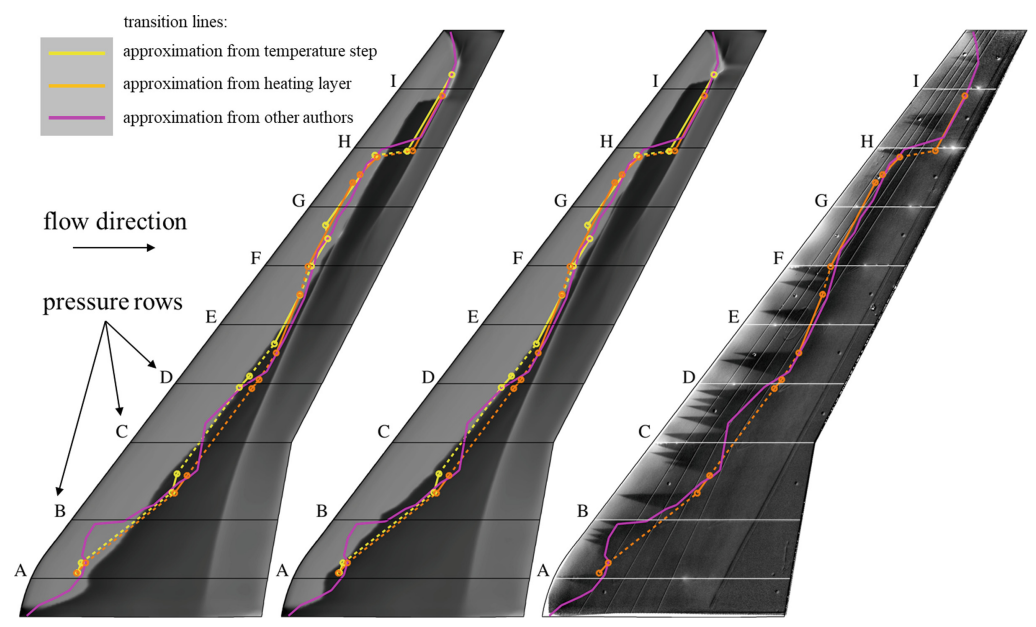

Fig. 4. CRM-NLF wing, comparison of predicted transition fronts, $\alpha=2 \cdot 0^{\circ}$. Left: RANS surface $\mathrm{c}_{\mathrm{f}}$-distribution, incompressible LST. Middle: RANS surface $\mathrm{c}_{\mathrm{f}}$-distribution, compressible LST. Right: TSP image, heating layer method.

Despite the visible minor differences in predicted transition locations among each other, the overall agreement of both prediction methods compared to the experimental data is very good. The most crucial deviation to the experiment can be seen at the outboard area of the wing for $\alpha=2.0^{\circ}$ (Fig. 4). Here transition is predicted too far upstream compared to the experiment between pressure rows $\mathrm{H}$ and I. Transition has been found to react in this region very sensibly to pressure gradient and shock location so that small deviations in the flow conditions (Mach number, angle of attack) may have a rather large impact on the predicted transition locations. The same is true for the other remarkable deviation between simulation and experiment for $\alpha=2.5^{\circ}$ and $\alpha=3.0^{\circ}$ (Figs. 5 and 6). Again, at the outboard area of the wing (beyond pressure row F), the predicted transition locations strongly depend on an accurate representation of the actual 


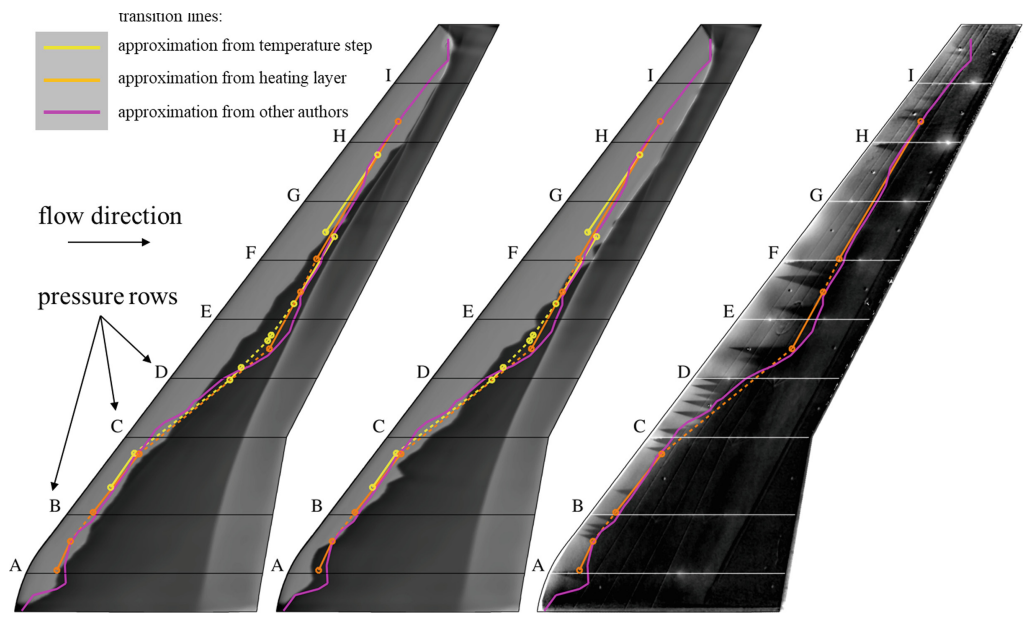

Fig. 5. CRM-NLF wing, comparison of predicted transition fronts, $\alpha=2.5^{\circ}$. Left: RANS surface $\mathrm{c}_{\mathrm{f}}$-distribution, incompressible LST. Middle: RANS surface $\mathrm{c}_{\mathrm{f}}$-distribution, compressible LST. Right: TSP image, heating layer method.

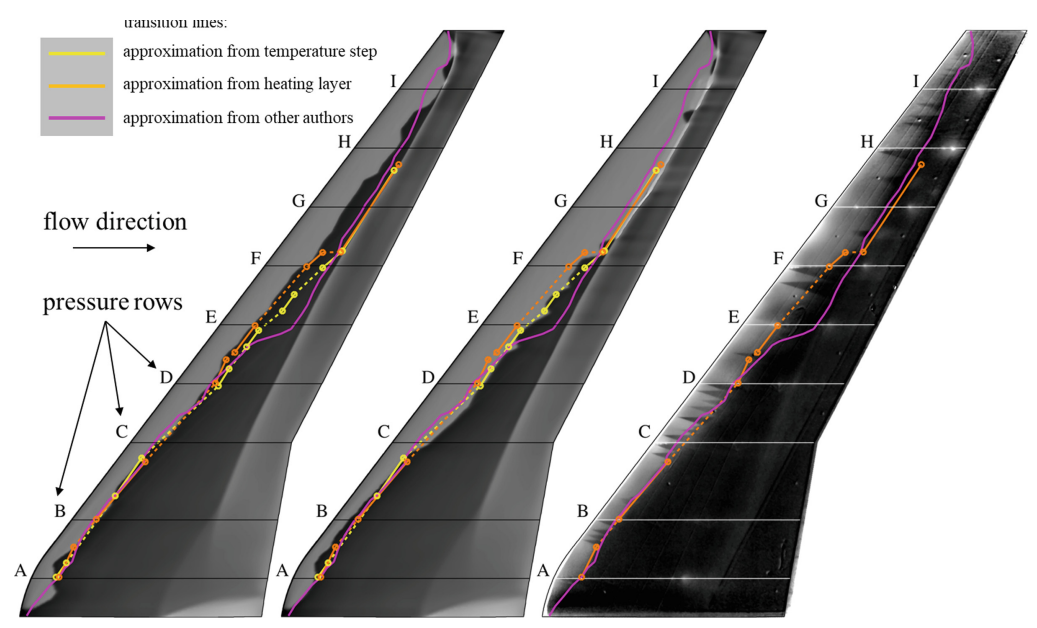

Fig. 6. CRM-NLF wing, comparison of predicted transition fronts, $\alpha=3.0^{\circ}$. Left: RANS surface $\mathrm{c}_{\mathrm{f}}$-distribution, incompressible LST. Middle: RANS surface $\mathrm{c}_{\mathrm{f}}$-distribution, compressible LST. Right: TSP image, heating layer method.

flow conditions and small deviations in Mach number or angle of attack may lead to a rather significant impact on the predicted transition lines.

An indication of a somewhat improper representation of the actual flow conditions in the wind tunnel during the numerical simulation can be obtained from the pressure distributions. Comparing numerically predicted pressure distributions for fully turbulent computations and computations with transition prediction with incompressible stability analysis (note: incompressible and compressible stability analysis give very similar transition locations and hence very similar pressure distributions) reveals, that generally the 

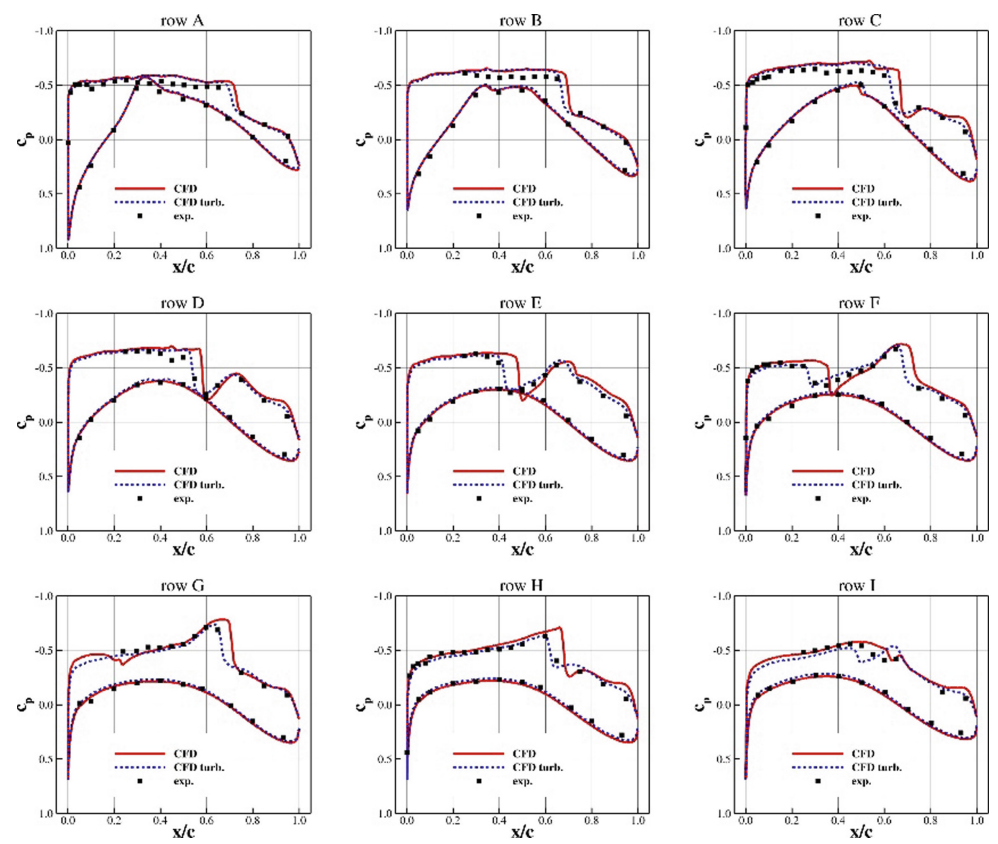

Fig. 7. CRM-NLF wing, pressure distribution at measuring sections, $\alpha=1.5^{\circ}$.

experimental pressure distribution is better matched by the fully turbulent computations for all considered cases (shown here only for $\alpha=1.5^{\circ}$ and $\alpha=3.0^{\circ}$ in Figs. 7 and 8 ). For the majority of angles of attack and pressure rows, the shock locations in the transitional computations are located too far downstream compared to the experiment. However, the pressure distributions of the fully turbulent computations are in very good agreement with the experiment. An explanation for this behavior, beyond a possible deviation in flow conditions applied in the computations compared to the experiment, may be seen in the larger amount of turbulent flow in the experiments. For all angles of attack considered here, a large amount of turbulent wedges forms in the experiment (Figs. 3, 4, 5 and 6, right image), leading to a different thickening of the boundary layer compared to laminar flow and thus having a possible impact on the location of the shock and hence on the pressure distribution. Including the turbulent wedges in the numerical computation has already led to an improved prediction of the pressure distribution for transitional flow computations [7]. 

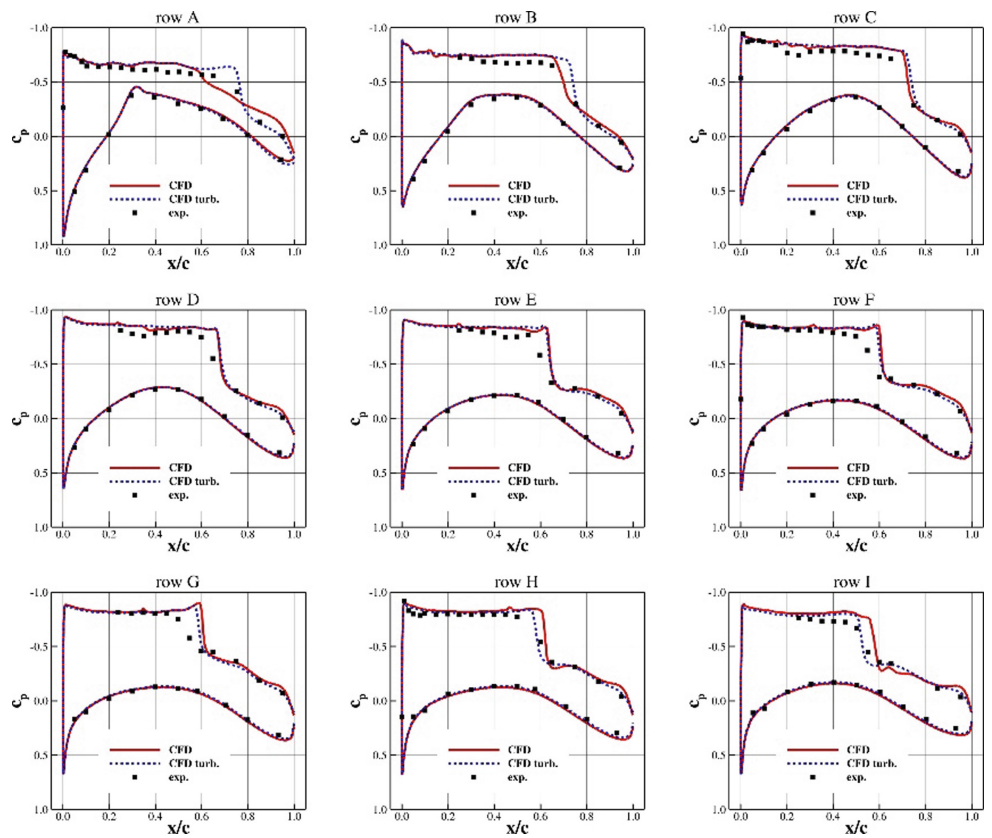

Fig. 8. CRM-NLF wing, pressure distribution at measuring sections, $\alpha=3.0^{\circ}$.

\section{Conclusion}

The determination of critical N-factors for the CRM-NLF wing revealed two different values for Tollmien-Schlichting instabilities, depending on the applied method. For an incompressible stability analysis a critical $\mathrm{N}$-factor of $\mathrm{N}_{\mathrm{TS}}=11.7$ was determined, whereas for a compressible stability analysis the critical $\mathrm{N}$-factor is $\mathrm{N}_{\mathrm{TS}}=4.1$. The latter is lower than the critical $\mathrm{N}$-factor applied in other investigations using compressible stability analysis but is just within the possible range determined for the facility accommodating the CRM-NLF experiments. The critical N-factor for cross-flow instabilities $\left(\mathrm{N}_{\mathrm{CF}}=6.0\right)$ is independent of applying incompressible or compressible analysis and corresponds to values used in other investigations.

Applying the critical $\mathrm{N}$-factors for transitional computations reveals a good agreement between predicted and experimental transition lines, respectively for incompressible and compressible stability analysis. Slight deviations in the numerical transition lines compared to the experiment may be explained by the sensitivity of the flow to the overall extent of turbulent flow (turbulent wedges in the experiment) and the accurate representation of the actual flow conditions of the experiment in the numerical simulation.

At this stage, the current results confirm the approach that for the prediction of the transition fronts incompressible LST as well as compressible LST can be applied leading to a similarly good agreement between the measured and predicted transition fronts as long as the correspondingly calibrated critical Tollmien-Schlichting N-factors are used. There are arguments in favor of incompressible LST over compressible LST [8] that 
need further discussion and investigation in the future and for the time being it seems reasonable to follow the established incompressible LST approach further on. On the other hand, it could be shown that in specific cases the application of compressible LST in the design process can lead to airfoil shapes that exhibit a larger laminar length and a lower wave drag [9]. Also in this respect, more work and investigations are imperative in the nearest future in order to achieve more data and knowledge for a better assessible situation.

Acknowledgement. The authors thank M. Fehrs of DLR for the provision of the computational grid and A. N. Watkins of NASA for the provision of heating layer and temperature step floating point TIFF images. The data source of the experimental pressure data is: commonresearchmodel.larc.nasa.gov/crm-nlf/.

\section{References}

1. Lynde, M.N., Campbell, R.L.: Computational Design and Analysis of a Transonic Natural Laminar Flow Wing for a Wind Tunnel Model. AIAA-2017-3058 (2017)

2. Lynde, M.N., Campbell, R.L., Viken, S.A.: Additional Findings from the Common Research Model Natural Laminar Flow Wind Tunnel Test. AIAA-2019-3292 (2019)

3. Watkins, A.N., Goodman, K.Z., Peak, S.M.: Transition Detection at Cryogenic Temperatures Using a Carbon-Based Resistive Heating Layer Coupled with Temperature Sensitive Paint. AIAA-2019-2191 (2019)

4. Krumbein, A., Krimmelbein, N., Schrauf, G.: Automatic transition prediction in hybrid flow solver, part 1: Methodology and sensitivities. J. Aircr. 46, 1176-1190 (2009)

5. Crouch, J., Sutanto, M., Witkowski, D., Watkins, A., Rivers, M., Campbell, R.: Assessment of the National Transonic Facility for Natural Laminar Flow Testing. AIAA-2010-1302 (2010)

6. Helm, S.: Private communication (2020)

7. Helm, S., Fehrs, M., Krimmelbein, N., Krumbein, A.: Transition Prediction and Analysis of the CRM-NLF wing with the DLR TAU-Code. To be published (2021)

8. Schrauf, G., Streit, T.: N-factors and design. Are we expecting too much? In: Presentation Charts, $1^{\text {st }}$ AIAA Transition Modelling and Prediction Workshop, 21-22 January 2021, Virtual Event.

9. Streit, T., Schrauf, G., Hein, S., Seitz, A., Kunze, P.: NLF-Potential für transsonische Langstreckenflugzeuge, Institutskolloquium AS-BS 3. DLR - Institut für Aerodynamik und Strömungstechnik, Braunschweig-Göttingen (Feb. 2021) 